\title{
Evaluation of Oxidative Stress and Antioxidant Status in Patients with Cardiovascular Disease in Rural Populations of the Nilgiris, South India
}

\author{
E. P. Kumar, ${ }^{1}$ Radhika Mukherjee, ${ }^{1}$ R. Senthil, ${ }^{2}$ S. Parasuraman, ${ }^{1}$ and B. Suresh ${ }^{1}$ \\ ${ }^{1}$ Department of Pharmacology, JSS College of Pharmacy, Ootacamund 643001, India \\ ${ }^{2}$ Department of Pharmacy Practise, JSS College of Pharmacy, Ootacamund 643001, India \\ Correspondence should be addressed to E. P. Kumar, premkumar1957@gmail.com
}

Received 29 November 2011; Accepted 20 December 2011

Academic Editors: G. M. Campo and R. Fantozzi

Copyright (C) 2012 E. P. Kumar et al. This is an open access article distributed under the Creative Commons Attribution License, which permits unrestricted use, distribution, and reproduction in any medium, provided the original work is properly cited.

Objective. The objective of this work was to study the risk factors of ischaemic heart disease (IHD) in rural populations of the Nilgiris, south India, with stress on the various social habits and oxidant stress. Methods. A total of 72 patients with cardiovascular disease (CVD) and 12 healthy volunteers were screened. Forty-seven patients with CVD (intervention group) and 10 healthy volunteers (control group) were randomly selected for the study. Written informed consent was obtained from all the participants, and their demographic details were collected. A $6 \mathrm{~mL}$ blood sample was collected from each of the participants, and the serum was separated in the samples. The levels of enzymic (superoxide dismutase, catalase) and nonenzymic antioxidants (ascorbic acid) in the plasma were determined biochemically. The level of thiobarbituric acid species (TBARS), which is a predictor of lipid peroxidation, was measured. Results. The participants of the study were stratified as according to demographic and social variables. The values of all the antioxidants and TBARS were statistically compared. Significantly reduced antioxidant levels and increased TBARS levels were found in the intervention group compared with the control group. The results suggest that the lowered antioxidant level may be a result of the oxidant stress of the disease. Statistically significant differences were not found in the antioxidant and TBARS levels when comparing smokers versus nonsmokers, alcoholics versus nonalcoholics, and vegetarians versus nonvegetarians. Conclusion. The major causes of CVD amongst the rural populations of the Nilgiris, south India, are preventable causes such as smoking and high fat intake, all of which cause oxidative stress, as seen in our study through various serum markers.

\section{Introduction}

India has one of the highest burdens of cardiovascular diseases (CVDs). The prevalence of cardiovascular disease (CVD) in rural India is $7.4 \%$, and in urban India, it is $11 \%$ [1]. The incidence of ischaemic heart disease (IHD) and coronary heart disease (CHD) is steadily increasing in the Indian subcontinent. According to World Health Report 2002, CVD will be the largest cause of death in India by 2020. It is predicted that 2.6 million Indians will die due to CHD. This number will represent $54.1 \%$ of all CVD deaths in the age group of 30-69 years [2]. The incidence of CHD in urban India is increasing steadily at a rapid pace. Although many studies have been carried out in urban populations, very few studies have examined the etiologic factors in rural Indian populations. IHD is a progressive disease arising when the supply of oxygen in the myocardium is compromised by impeded blood flow in the coronary vasculature caused by luminal occlusion. IHD leads to the development of atherosclerosis and hypertrophy of musculature. The pathogenesis of atherosclerosis involves damage to the capillary endothelium caused by various factors including oxidized low-density lipoprotein (LDL) $[3,4]$. Muscular hypertrophy is caused by elaboration of local elaboration of growth factors from surrounding macrophages which are primed by free radicals. The plaque formed thereupon is covered by thinned out intima and media. A luminal compromise of nearly $70 \%$ is needed to produce symptomatic IHD. Free radicals are chemical species that can be considered as fragments of molecules possessing unpaired electrons. They are generally very reactive [5], and they are produced continuously in cells either as 
by-products of metabolism or during phagocytosis [6]. The major types of free radicals found in biological systems are reactive oxygen species (ROS) and reactive nitrogen species (RNS) [7]. Free radicals are produced mainly by cellular and environmental sources. They are generated by absorption of radiant energy, endogenous oxidative reactions, enzymatic metabolism of exogenous chemicals, and generation of free radicals in pathogenic conditions [8]. The present study was planned and conducted to evaluate the role of oxidative stress in patients with CVD patients from populations of the Nilgiris, south India, and the role of antioxidants in patients with IHD patients and to assess the classical risk factors associated with the oxidative stress related to CVD.

\section{Materials and Methods}

An open randomized control study was conducted at the Government District Headquarters Hospital, Ootacamund, Nilgiris District, Tamil Nadu, India, over 10 months. A total of 72 patients with CVD and 12 healthy volunteers were screened for the study. The study was carried out for 10 months from May 2005 to February 2006. The study was approved by the Institutional Ethics Committee, J.S.S. College of Pharmacy, Ootacamund, and the study was performed according to the recommendations of the Declaration of Helsinki.

The study was conducted on patients (31-80 years) suffering from CVD, such as, IHD, myocardial infarction (MI) or unstable angina (UA), who were admitted in the Intensive Cardiac Care Unit (ICCU)/Intensive Care Unit (ICU) of the Government District Headquarters, Ootacamund. IHD patients with chronic diseases such as diabetes and hypertension and those who had suffered strokes were excluded. Pregnant women and lactating mothers were also excluded from the study.

Forty-seven patients in the 31-80 year age group and 10 healthy volunteers in the 31-40 year age group were included in the intervention and control groups, respectively. These subjects were selected randomly without any sex predisposition. The subjects or their guardians were interviewed for 15-20 minutes, and their demographic details were collected. The demographic details included primary patient information (such as name, age, and sex), socioeconomic status and food habituation. After enrolment as a study participants, the history was collected through a structured enrolment form, the medical records (case history of patients) which included the age, gender, inpatient number, socioeconomic status, social habits, past medical, and surgical history.

A $6 \mathrm{~mL}$ fasting blood sample was collected from each participant. The blood samples were centrifuged at 3000 RPM for 20 minutes, and the serum was separated and stored at $-20^{\circ} \mathrm{C}$ until analysis. The levels of enzymic (superoxide dismutase (SOD) [9] and catalase (CAT) [10]) and nonenzymic (ascorbic acid [11]) antioxidants and predictor of lipid peroxidation as measured by the thiobarbituric acid species (TBARS) [12] in the serum were determined. Flow diagram of requirement and allocation of participants and analysis of the data are given in Figure 1.
The mean \pm SEM values were calculated for each group. Significant difference between groups was determined using one-way ANOVA followed by the Bonferroni test. A $P$ value less than 0.05 was considered as significant.

\section{Results}

Including the healthy controls 57 subjects were enrolled in the study. The greatest number of subjects was in the $61-70$ year group (20-of which 13 were males and 7 were females) and 51-60 year group (16-of which 9 were males and 7 were females). In all, there were 33 male subjects (58\%) and 24 female subjects $(42 \%)$ in the intervention group (Table 1).

In our study, 21/30 males and 9/17 females had a BMI of $21-25 \mathrm{~kg} / \mathrm{m}^{2}$. No patient had a BMI greater than 30 . Thus none of the patients in the study had obesity as a predisposing factor. Of the 47 patients, 33 were workers with an average monthly income of Rs.600. In the intervention group, 26 male subjects out of 30 and 7 female subjects out of 17 were workers by occupation. The prevalence of CVD was highest in this group. This may be due to their habits, such as heavy smoking and having a high alcohol intake and high consumption of animal fat. In the control group, most of the subjects were vegetarians, nonsmokers and nonalcoholic subjects. Most of the male subjects in the intervention group were nonvegetarians, smokers, and alcoholics (Table 2).

The levels of enzymic and nonenzymic antioxidants of the patients with CVD were evaluated after the initial symptomatic management was done. The mean value of SOD levels in healthy participants was found to be $2.15 \pm 0.01$ units/minute/mg protein. In patients with CVD, a significant reduction $(P<0.001)$ in SOD levels was observed, compared with healthy control participants. The SOD levels observed in patients with IHD, MI, and UA were $1.05 \pm 0.01,1.54 \pm$ 0.01 and $1.86 \pm 0.01$, units/minute/mg protein, respectively (Table 3).

The mean value of catalase in healthy participants was found to be $19.33 \pm 0.81 \mu$ moles of $\mathrm{H}_{2} \mathrm{O}_{2}$ consumed/minute/mg protein. In patients with $\mathrm{CVD}$, a significant reduction $(P<0.001)$ in the catalase values was observed compared with healthy control participants. The catalase levels observed in patients with IHD, MI, and UA were $6.01 \pm 0.45,6.73 \pm 0.14$, and $6.28 \pm 0.08 \mu$ moles of $\mathrm{H}_{2} \mathrm{O}_{2}$ consumed/minute/mg protein, respectively.

The normal mean value of ascorbic acid was found to be $139.20 \pm 10.08 \mathrm{mg} / \mathrm{dL}$. In patients with CVD, a significant reduction $(P<0.001)$ in the ascorbic acid values was observed when compared with the control participants. The ascorbic acid levels observed in patients with IHD, MI, and UA were $66.60 \pm 17.32,57.92 \pm 10.17$, and $71.90 \pm$ $9.73 \mathrm{mg} / \mathrm{dL}$, respectively. The estimated level of antioxidants in the intervention group was lower than that in the control group. The results of the present investigation suggest that the lowered level of antioxidants may be a result of the oxidative stress of the disease. Individual comparisons of ascorbic acid levels showed that the differences between smoker versus nonsmokers, alcoholics versus nonalcoholics and vegetarians versus nonvegetarians were not statistically significant (Tables 4,5 , and 6 ). 


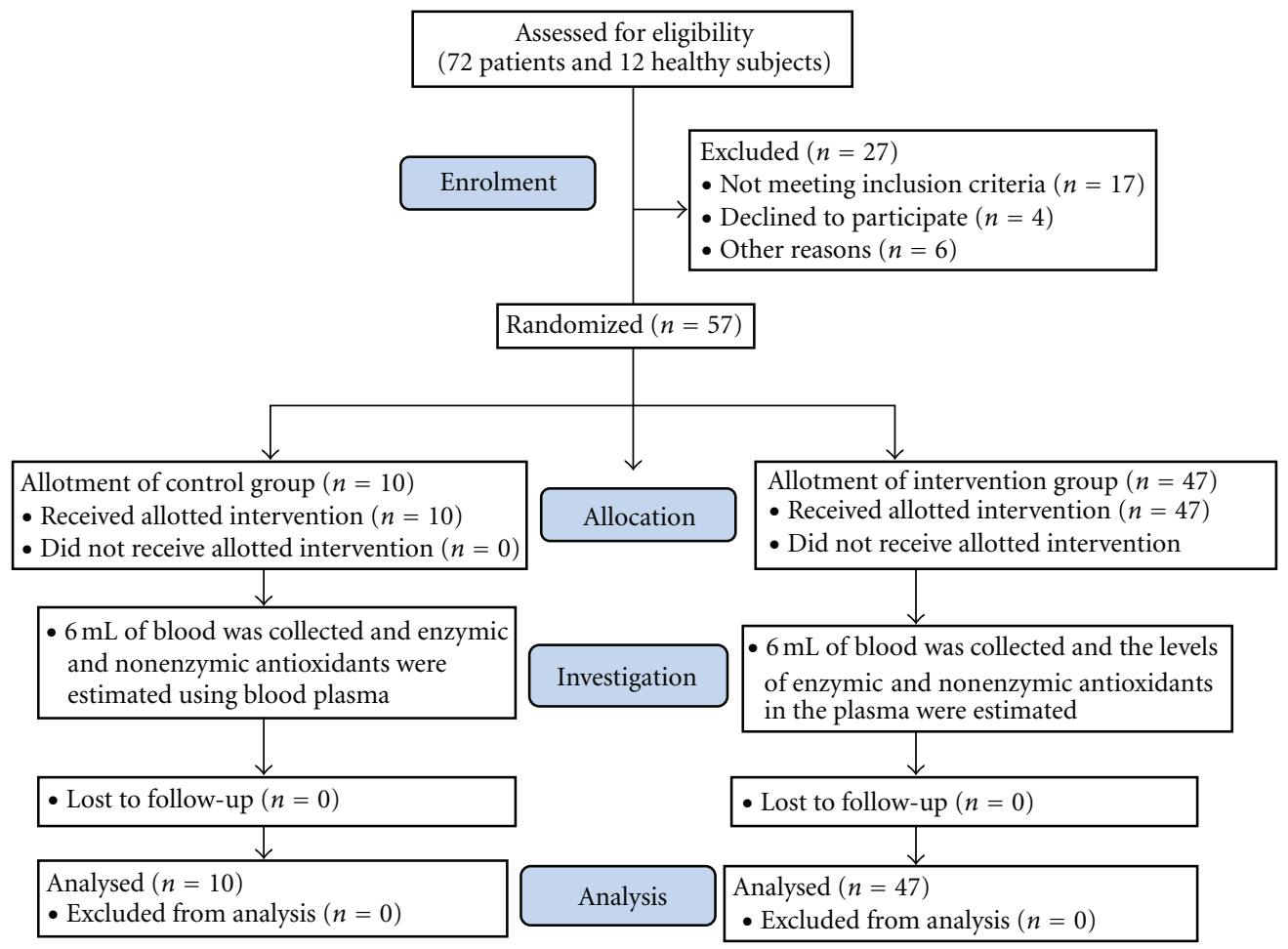

FIgURE 1: Requirement, allocation, and follow-up of participants.

TABLE 1: Categorization of subjects based on age group.

\begin{tabular}{|c|c|c|c|c|c|c|c|c|}
\hline \multirow{4}{*}{ Age group (years) } & \multicolumn{6}{|c|}{ Subjects } & & \\
\hline & \multirow{2}{*}{\multicolumn{2}{|c|}{ Control group (normal) }} & \multicolumn{6}{|c|}{ Intervention group } \\
\hline & & & \multicolumn{2}{|c|}{ IHD } & \multicolumn{2}{|c|}{ MI } & \multicolumn{2}{|c|}{ UA } \\
\hline & Male & Female & Male & Female & Male & Female & Male & Female \\
\hline $31-40$ & 3 & 7 & 2 & 0 & 1 & 0 & 0 & 0 \\
\hline $41-50$ & 0 & 0 & 2 & 1 & 2 & 0 & 0 & 1 \\
\hline $51-60$ & 0 & 0 & 4 & 3 & 1 & 1 & 4 & 3 \\
\hline $61-70$ & 0 & 0 & 7 & 6 & 5 & 0 & 1 & 1 \\
\hline $71-80$ & 0 & 0 & 0 & 0 & 1 & 1 & 0 & 0 \\
\hline Total & \multicolumn{2}{|c|}{10} & \multicolumn{2}{|c|}{25} & \multicolumn{2}{|c|}{12} & \multicolumn{2}{|c|}{10} \\
\hline
\end{tabular}

IHD: ischaemic heart disease; MI: myocardial infarction; UA: unstable angina.

TABLE 2: Categorization of subjects based on social habits.

\begin{tabular}{|c|c|c|c|c|c|c|}
\hline \multirow{2}{*}{ Gender } & \multicolumn{2}{|c|}{ Diet } & \multicolumn{2}{|c|}{ Smoking habits } & \multicolumn{2}{|c|}{ Alcohol intake } \\
\hline & Vegetarian & Nonvegetarian & Smoking & Nonsmoking & Alcoholic & Nonalcoholic \\
\hline \multicolumn{7}{|l|}{ Male } \\
\hline $\mathrm{N}$ & 3 & 0 & 0 & 3 & 0 & 3 \\
\hline CVD & 1 & 29 & 28 & 2 & 12 & 18 \\
\hline \multicolumn{7}{|l|}{ Female } \\
\hline $\mathrm{N}$ & 7 & 0 & 0 & 7 & 0 & 7 \\
\hline CVD & 4 & 13 & 0 & 17 & 0 & 17 \\
\hline Total & 15 & 42 & 28 & 29 & 12 & 45 \\
\hline
\end{tabular}

N: normal; CVD: cardiovascular disease. 
TABLE 3: Levels of antioxidants and lipid peroxidase in healthy control and patients with CVD.

\begin{tabular}{lcccc}
\hline Subjects enrolled & SOD & Catalase & $\begin{array}{c}\text { TBARS } \\
(\mu \text { moles of } \\
\text { malondialdehyde/dL } \\
\text { serum })\end{array}$ & $\begin{array}{c}\text { Ascorbic acid } \\
(\mathrm{U} / \mathrm{mg} \text { protein })\end{array}$ \\
\hline Normal $(n=10)$ & $2.15 \pm 0.01$ & $19.33 \pm 0.81$ & $68.70 \pm 6.73$ & $139.20 \pm 10.08$ \\
IHD $(n=25)$ & $1.05 \pm 0.01^{* * *}$ & $6.01 \pm 0.45^{* * *}$ & $170.16 \pm 41.12$ & $66.60 \pm 17.32^{*}$ \\
MI $(n=12)$ & $1.54 \pm 0.01^{* * *}$ & $6.73 \pm 0.14^{* * *}$ & $204.92 \pm 24.52$ & $57.92 \pm 10.17^{*}$ \\
UA $(n=10)$ & $1.86 \pm 0.01^{* * *}$ & $6.28 \pm 0.08^{* * *}$ & $170.70 \pm 19.23$ & $71.90 \pm 9.73$ \\
\hline
\end{tabular}

IHD, ischaemic heart disease; MI, myocardial infarction; UA, unstable angina.

Values are mean \pm SEM.

${ }^{*} P<0.05 ; * * * P<0.001$ compared with control. One-way ANOVA followed by Bonferroni test.

TABLE 4: Levels of antioxidants and lipid peroxidase in patients ischaemic heart disease based on social habits.

\begin{tabular}{lcccc}
\hline Social habits & SOD & Catalase & $\begin{array}{c}\text { TBARS } \\
(\mu \text { moles of }\end{array}$ & $\begin{array}{c}\text { Ascorbic acid } \\
\text { malondialdehyde/dL of } \\
\text { serum })\end{array}$ \\
\hline Smoker $(n=18)$ & $1.03 \pm 0.03$ & $6.077 \pm 0.44$ & $181.53 \pm 35.51$ & $66.27 \pm 20.54$ \\
Nonsmoker $(n=7)$ & $1.11 \pm 0.05$ & $5.90 \pm 0.47$ & $156.70 \pm 42.80$ & $67.00 \pm 11.98$ \\
Alcoholic $(n=6)$ & $1.02 \pm 0.05$ & $5.79 \pm 0.40$ & $176.83 \pm 41.24$ & $60.66 \pm 12.07$ \\
Nonalcoholic $(n=19)$ & $1.01 \pm 0.01$ & $6.08 \pm 0.45$ & $168.05 \pm 41.97$ & $68.42 \pm 18.55$ \\
Vegetarian $(n=2)$ & $1.18 \pm 0.01$ & $6.35 \pm 0.49$ & $198.51 \pm 23.33$ & $53.51 \pm 12.12$ \\
Nonvegetarian $(n=23)$ & $1.04 \pm 0.04$ & $5.98 \pm 0.46$ & $167.69 \pm 41.71$ & $67.69 \pm 17.61$ \\
\hline
\end{tabular}

Values are mean \pm SEM.

TABLE 5: Levels of antioxidants and lipid peroxidase in patients with myocardial infarction according to social habits.

\begin{tabular}{lcccc}
\hline Social habits & SOD & Catalase & $\begin{array}{c}\text { TBARS } \\
(\mu \text { moles of } \\
\text { malondialdehyde/dL of } \\
\text { serum })\end{array}$ & $\begin{array}{c}\text { Ascorbic acid } \\
(\mathrm{mg} / \mathrm{dL})\end{array}$ \\
\hline Smoker $(n=6)$ & $1.50 \pm 0.02$ & $6.68 \pm 0.17$ & $214.16 \pm 22.60$ & $51.83 \pm 11.05$ \\
Nonsmoker $(n=6)$ & $1.56 \pm 0.01$ & $6.77 \pm 0.10$ & $195.62 \pm 24.50$ & $64.00 \pm 14.04$ \\
Alcoholic $(n=4)$ & $1.49 \pm 0.02$ & $6.72 \pm 0.22$ & $210.58 \pm 25.60$ & $53.75 \pm 11.61$ \\
Nonalcoholic $(n=8)$ & $1.56 \pm 0.02$ & $6.73 \pm 0.10$ & $202.11 \pm 22.10$ & $60.00 \pm 9.47$ \\
Vegetarian $(n=1)$ & $1.60 \pm 0.03$ & $6.58 \pm 0.12$ & $186.00 \pm 20.20$ & $55.01 \pm 12.32$ \\
Nonvegetarian $(n=11)$ & $1.53 \pm 0.03$ & $6.74 \pm 0.14$ & $206.62 \pm 24.90$ & $58.18 \pm 10.62$ \\
\hline
\end{tabular}

Values are mean \pm SEM.

TABLE 6: Levels of antioxidants and lipid peroxidase in patients with unstable angina according to social habits.

\begin{tabular}{|c|c|c|c|c|}
\hline \multirow{2}{*}{ Social habits } & SOD & Catalase & TBARS & Ascorbic acid \\
\hline & (U/mg protein) & (U/mg protein) & $\begin{array}{c}(\mu \text { moles of } \\
\text { malondialdehyde/dL of } \\
\text { serum })\end{array}$ & $(\mathrm{mg} / \mathrm{dL})$ \\
\hline Smoker $(n=4)$ & $1.82 \pm 0.01$ & $6.23 \pm 0.07$ & $188.25 \pm 8.62$ & $74.25 \pm 13.45$ \\
\hline Nonsmoker $(n=6)$ & $1.88 \pm 0.02$ & $6.31 \pm 0.07$ & $159.00 \pm 14.50$ & $70.33 \pm 7.39$ \\
\hline Alcoholic $(n=2)$ & $1.82 \pm 0.01$ & $6.28 \pm 0.05$ & $182.00 \pm 17.07$ & $80.5 \pm 4.94$ \\
\hline Nonalcoholic $(n=8)$ & $1.86 \pm 0.03$ & $6.28 \pm 0.09$ & $157.87 \pm 20.55$ & $69.75 \pm 9.58$ \\
\hline Vegetarian $(n=2)$ & $1.90 \pm 0.01$ & $6.37 \pm 0.02$ & $177.50 \pm 16.26$ & $85.50 \pm 5.14$ \\
\hline Nonvegetarian $(n=8)$ & $1.84 \pm 0.02$ & $6.26 \pm 0.07$ & $169.00 \pm 20.52$ & $68.50 \pm 7.42$ \\
\hline
\end{tabular}

Values are mean \pm SEM. 
The normal mean value of TBARS was found to be $68.70 \pm 6.73 \mu$ moles of malondialdehyde/dL serum. Increased levels of TBARS were observed in subjects with IHD, MI, and UA, but the results were not statistically significant. The increased TBARS levels may be a result of the oxidant stress of the disease. Individual comparisons of the TBARS level between smokers versus nonsmokers, alcoholics, versus nonalcoholics and vegetarians versus non-vegetarians showed that the differences were not statistically significant (Tables 4,5 , and 6).

\section{Discussion}

To evaluate the oxidative stress and antioxidant status in patients with CVD in a rural Indian population, a crosssectional study was carried out in which an intervention group consisting of 47 patients with CVD was compared with 10 healthy volunteers.

The demographic data showed a higher incidence of IHD in males compared with females of the same age group. The lower incidence in menopausal and postmenopausal females is probably due to the protective effect of estrogens. Obesity was not an important risk factor, as seen in our study. The presence of other comorbid factors may be responsible for the oxidant damage caused due to smoking and diabetes. Earlier studies have suggested that, in both men and women, diabetes, age, and plasma cholesterol levels were independent predictors of IHD mortality. The prevalence of IHD and relative mortality risk associated with several risk factors (age, marital status, education, cigarette smoking, systolic blood pressure, cholesterol, fasting plasma glucose, and obesity) differ with sex $[13,14]$.

When the mean values of the serum enzymes were compared, a marked decrease in the levels of SOD and catalase was found, whereas the level of TBARS, indicating lipid peroxidation, was markedly increased. Evaluation of the serum values in various demographic subsets of the population was performed, and the greatest changes were found in smokers, followed by nonvegetarians, suggesting that smoking has an important role in causing oxidant stress.

Free radicals, cytokines, nitric oxide (NO), and antioxidants play a major role in both atherosclerosis and myocardial damage and preservation [15]. In the present study, it was found that rural people from the Nilgiris admitted with IHD, MI, and UA had increased TBARS levels and reduced SOD, catalase, and ascorbic acid levels. Previous studies have demonstrated the biological relevance of the antioxidant defence system to myocardial infarction. Chandra et al. (1994) demonstrated that there were elevated superoxide anion, malonyldialdehyde, and glutathione reductase levels and reduced superoxide dismutase and catalase levels in unstable angina and acute myocardial infarction cases [16]. Gupta and Chari (2006) measured the levels of proxidants and antioxidants in patients with IHD and type II diabetes mellitus. Significantly increased levels of malondialdehyde and decreased levels of superoxide dismutase, glutathione peroxidase, and vitamin $\mathrm{C}$ were found in diabetics without complications and nondiabetics with IHD compared with control subjects [17].
The levels of SOD, catalase, and ascorbic acid were shown to be lower and the TBARS level was shown to be higher in patients from rural India with IHD, MI, and unstable angina. Risk factors such as age, being male, smoking, alcohol consumption, a nonvegetarian diet, and occupation played an important role in the genesis of oxidative stress, which is one of the causes of CVD.

\section{Conclusion}

It is concluded on the basis of the present study that free radicals are implicated in the etiology of CVD and that enzymatic antioxidants, SOD, and catalase have a major role in preventing cellular oxidative damage.

\section{References}

[1] M. D. Huffman, D. Prabhakaran, C. Osmond et al., "Incidence of cardiovascular risk factors in an Indian urban cohort: results from the New Delhi Birth Cohort," Journal of the American College of Cardiology, vol. 57, no. 17, pp. 1765-1774, 2011.

[2] National Cardiovascular Disease Database (Sticker No: SE / 04 / 233208), Supported by Ministry of Health \& Family Welfare, Government of India and World Health Organization, 2011, http://www.whoindia.org/.

[3] R. Ross, "The pathogenesis of atherosclerosis: a perspective for the 1990s," Nature, vol. 362, no. 6423, pp. 801-809, 1993.

[4] H. Shimokawa, "Primary endothelial dysfunction: atherosclerosis," Journal of Molecular and Cellular Cardiology, vol. 31, no. 1, pp. 23-37, 1999.

[5] G. Ray and S. A. Husain, "Oxidants, antioxidants and carcinogenesis," Indian Journal of Experimental Biology, vol. 40, no. 11, pp. 1213-1232, 2002.

[6] J. Chen and J. L. Mehta, "Role of oxidative stress in coronary heart disease," Indian Heart Journal, vol. 56, no. 2, pp. 163173, 2004.

[7] M. Irshad and P. S. Chaudhuri, "Oxidant-antioxidant system: role and significance in human body," Indian Journal of Experimental Biology, vol. 40, no. 11, pp. 1233-1239, 2002.

[8] M. Ajitha and K. Rajnarayana, "Role of oxygen free radicals in human disease," Indian Drugs, vol. 38, no. 11, pp. 545-554, 2001.

[9] P. Kakkar, B. Das, and P. N. Viswanathan, "A modified spectrophotometric assay of superoxide dismutase," Indian Journal of Biochemistry and Biophysics, vol. 21, no. 2, pp. 130132, 1984.

[10] R. F. Beers and I. W. Sizer, "A spectrophotometric method for measuring the breakdown of hydrogen peroxide by catalase," The Journal of Biological Chemistry, vol. 195, no. 1, pp. 133$140,1952$.

[11] E. J. Hewitt and G. J. Dickes, "Spectrophotometric measurements on ascorbic acid and their use for the estimation of ascorbic acid and dehydroascorbic acid in plant tissues," The Biochemical Journal, vol. 78, pp. 384-391, 1961.

[12] H. Ohkawa, N. Ohishi, and K. Yagi, "Reaction of linoleic acid hydroperoxide with thiobarbituric acid," Journal of Lipid Research, vol. 19, no. 8, pp. 1053-1057, 1978.

[13] D. L. Wingard, L. Suarez, and E. Barrett Connor, "The sex differential in mortality from all causes and ischemic heart disease," American Journal of Epidemiology, vol. 117, no. 2, pp. 165-172, 1983. 
[14] E. Barrett Connor and D. L. Wingard, "Sex differential in ischemic heart disase mortality in diabetics: a prospective population-based study," American Journal of Epidemiology, vol. 118, no. 4, pp. 489-496, 1983.

[15] U. N. Das, "Free radicals, cytokines and nitric oxide in cardiac failure and myocardial infarction," Molecular and Cellular Biochemistry, vol. 215, no. 1-2, pp. 145-152, 2000.

[16] M. Chandra, N. Chandra, R. Agrawal, A. Kumar, A. Ghatak, and V. C. Pandey, "The free radical system in ischemic heart disease," International Journal of Cardiology, vol. 43, no. 2, pp. 121-125, 1994.

[17] M. Gupta and S. Chari, "Proxidant and antioxidant status in patients of type II diabetes mellitus with IHD," Indian Journal of Clinical Biochemistry, vol. 21, no. 2, pp. 118-122, 2006. 

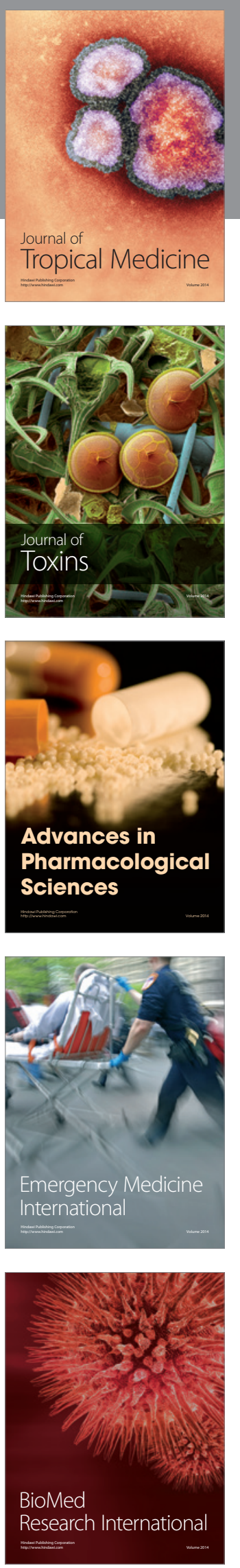
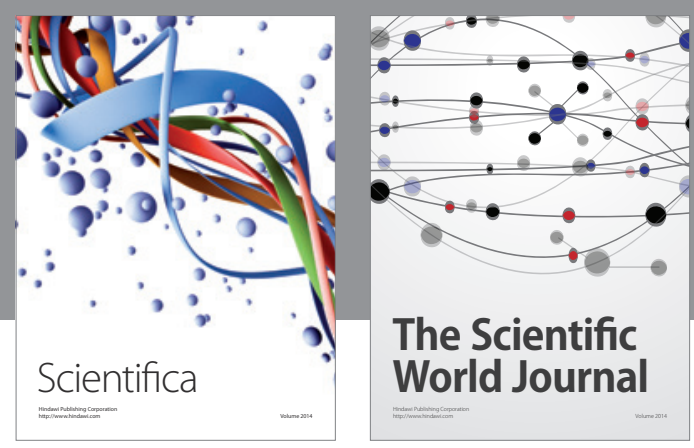

The Scientific World Journal
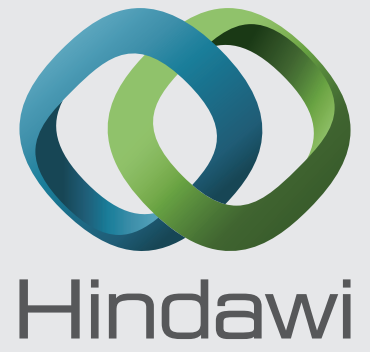

Submit your manuscripts at

http://www.hindawi.com
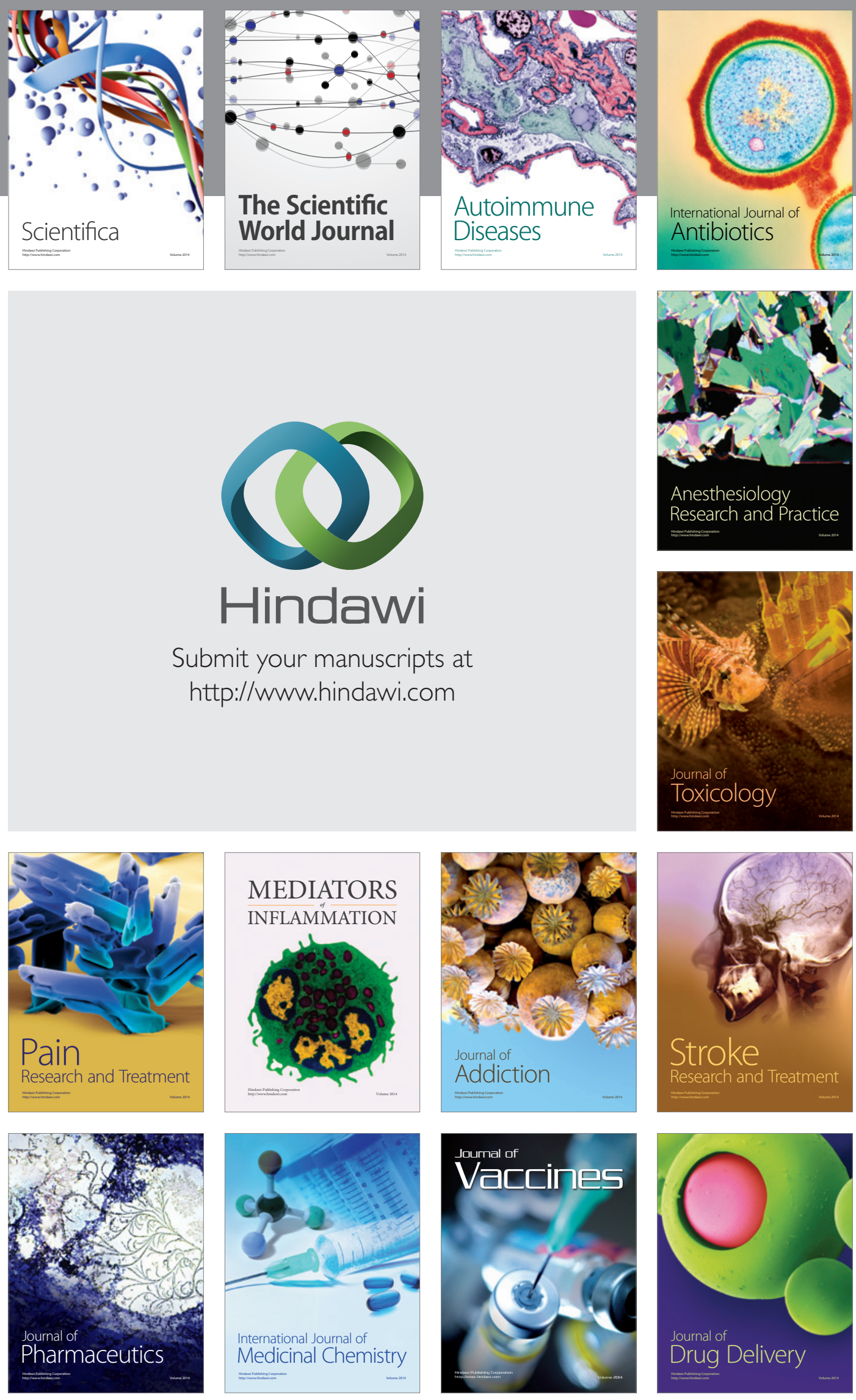